\title{
Potencial de cambio en las capacidades humanas de los docentes de educación básica en la región socioeducativa de Tuxtla Gutiérrez, Chiapas, México
}

\section{Potential change in human capacities of teachers of basic education in the socio-educational region of Tuxtla Gutierrez, Chiapas, Mexico}

\author{
Irán Guadalupe RODRÍGUEZ RAMÍREZ \\ Universidad Autónoma de Chiapas y Universidad de Málaga
}

Recibido: Noviembre 2014

Evaluado: Febrero 2015

Aceptado: Marzo 2015

\begin{abstract}
Resumen
La investigación que se presenta fue desarrollada dentro del campo de los estudios regionales y con visión transdisciplinar. Tuvo como objetivo deconstruir conjuntamente con los participantes, las capacidades humanas regionales de nosotros los docentes de educación básica, en nuestros procesos de reflexión entre teoría y práctica, en los que se consideraron las condicionantes estructurales, en niveles macro y micro regionales, con el alcance de haber identificado el potencial de cambio en nuestra praxis de docente como acción ante nuestros alumnos, quienes configuran el tejido social de Tuxtla Gutiérrez. Desde una perspectiva sociocrítica, se tomó como punto de fuga o núcleo la teoría crítica con fundamento filosófico en la escuela de Fráncfort y con una postura epistemológica basada en el construccionismo por la estrecha relación sujeto-objeto en el estudio; por ende se empleó la metodología investigaciónacción a través de diversas técnicas e instrumentos. Los resultados fueron concentrados en categorías que facilitaron su discusión: primeramente con el potencial de cambio, que adquirió significado mediante el desarrollo de las capacidades de reflexión y conciencia del docente; luego se presentaron las capacidades humanas, de nosotros los docentes, en las que destacaron como base de otras, las capacidades de amor, autoestima y compromiso. Posteriormente, se describió la reflexión docente sobre la concepción del beneficio personal hacia el beneficio colectivo. Finalmente, se discutió sobre la proyección de la acción docente dentro de la relación alumno y región. Se concluyó que las capacidades humanas y el potencial de cambio, deconstruidos conjuntamente con los docentes participantes, fueron la respuesta del proceso investigativo en un determinado tiempo y espacio, como posibilidades de acción docente hacia la tan deseada transformación socioeducativa regional.
\end{abstract}

Palabas clave: potencial de cambio, capacidades humanas, beneficio personal y colectivo, docente-alumno-región. 


\begin{abstract}
The research presented was developed within the field of regional studies with a transdisciplinary vision. It aimed to deconstruct together with the participants, the regional human capacities of the teachers of basic education, in our thought processes between theory and practice, where the structural conditions were considered, in macro and micro regional levels, with the scope of identifying the potential for change in our teaching praxis as an action for our students, who make up the social fabric of Tuxtla Gutierrez. From a socio-critical perspective the critical theory with philosophical foundation of the school of Frankfurt with an epistemological position based on constructionism through the narrow subject-object relationship was taken as vanishing point or core. Finally, action research methodology was used through various techniques and instruments. The results were concentrated in categories that facilitated the discussion: first, with the potential for change, which acquired significance by developing skills of reflection and awareness of teachers; then the human capacities of teachers, where capacities to love, self-esteem and commitment were stood out as the base of others. Later, teacher reflection was described based on the conception of personal benefit to collective benefit. Finally, the projection of the teaching activity within the relationship between region and student was discussed. It was concluded that human capabilities and the potential for change, deconstructed at the same time with participating teachers were the answer to the research process in a given time and space, as teaching possibilities towards the desired regional socio-educative transformation.
\end{abstract}

Keywords: potential for change, human capacities, personal and collective benefit, teacherlearner-region.

Los estudios regionales se pueden comprender como el producto de una imperante demanda de la sociedad actual, que inmersa en un mundo en el que ocurren diversos procesos culturales, educativos, económicos y políticos, por mencionar algunos, los cuales se desarrollan para el bienestar propio, no logran explicar las desigualdades entre las poblaciones de diferentes territorios. Esto nos lleva a reflexionar sobre los escritos de Touraine (2005), para comprender que no todos somos ciudadanos del mismo mundo en que se vive hoy, porque no es una unidad institucional y política, lo que conduce a una conciencia de existencia común pero a la vez de independencia, con la necesidad de reconocer que entre unos y otros se poseen historias propias que finalmente no son totalmente separadas.

La sociedad contemporánea está viviendo un momento histórico y común de transformación, porque de acuerdo a Ferrero (2006) se encuentra dentro de un sistema mundial en plena globalización; en este sentido, con base en autores como Beck (2008) y Bonal (2002), se puede concebir una nueva cultura entre lo global y lo local, entendida en un proceso dialéctico, por las formas de comunicación transculturales entre las poblaciones, que realizan movilidades de mercancías y de capitales, tanto monetarios como humanos, mediante estrategias económicas, políticas y mediáticas, que tienen el fin de aumentar las tasas de beneficio, aunque en la realidad parecieran ser contradictorias.

Estas concepciones implican en los estudios regionales, trabajar en propuestas diferenciadas como lo propone Martínez - Pellégrini (2003), porque ante tantas 
desigualdades sociales, es necesario aceptar que una región posee una mezcla específica de características, la cual lleva a identificar el problema regional, para luego explicarlo y llegar a una solución, que finalmente orientará la trayectoria de su desarrollo, considerado este último concepto, para Sánchez Juárez (2011), como un proceso complejo entre lo exógeno y endógeno, que mediante el potencial social, económico y territorial de una región, se logra alcanzar una mejor condición o situación.

Ante lo descrito anteriormente, el propósito de este artículo es el dar a conocer los resultados de la investigación educativa desarrollada de manera participativa, dentro de un campo de conocimiento que se potencia ante los problemas de nuestro siglo y que desde "afuera" no se logran resolver. Dirigir la mirada hacia una región, implica entonces tomar en cuenta las fuerzas exógenas y endógenas que convergen en ella, así como todas las características, componentes y condicionantes que posee, sin olvidar que se construye dicha región en función del objeto de estudio (Ixtacuy, 2012).

Es por ello que el objeto de estudio de la investigación educativa que se presenta son las capacidades humanas en los procesos de reflexión del docente de educación básica, que se identifican por medio de la micro-acción dentro la región socioeducativa en Tuxtla Gutiérrez; esa región interna se compone de elementos objetivos tangibles y nociones subjetivas intangibles, determinadas por la estructura y la acción: Capacitadores, docentes, procesos de enseñanza-aprendizaje y por supuesto las capacidades humanas.

\section{Justificación del estudio}

A partir de los planteamientos de Max-Neef (2004) inicia un árbol de problemas, porque él considera una serie de problemáticas de impacto global y efectos a largo plazo, como la pobreza, crisis ambientales y destrucción de tejidos sociales, que vistas como fenómenos integrales se convierten en desafíos claramente transdisciplinarios. La pobreza puede ser reducida, de acuerdo a la propuesta de Mc Cauley (2006), por medio de estrategias encaminadas al desarrollo del capital humano, refiriéndose a la educación básica como factor clave que promueve enfoques de desarrollo incluyente. En ese sentido Sen (2000) propone una perspectiva más amplia para intervenir en dichas problemáticas, con la expansión de la capacidad humana, para tener libertades de agencia y bienestar, traducidos en desarrollo humano, refiriéndose también al logro de este mediante la educación básica.

Con estos planteamientos, la educación básica en la sociedad es sin duda una importante fuente de posibilidades de cambio ante las problemáticas del siglo XXI; esto lleva la mirada a los diversos actores que intervienen en los procesos educativos, de los que destaca el docente por ser quien forma a alumnos que configuran el tejido social de una región. Este actor resalta puesto que uno de los problemas del desarrollo profesional docente, considerado como reto actual de la investigación educativa, podría resolverse mediante la reflexión de las propias capacidades humanas: "De Miguel et. al. (1996)...[afirma que] se concede poca importancia a la autoobservación y la 
reflexión personal. El profesorado no ha sido formado para ello. Su función docente se centra fundamentalmente en el trabajo de aula" (Sandín, 2004, p. 23).

Con todo lo anterior, adquieren relevancia dos aspectos directamente relacionados: primero, las capacidades humanas de los docentes, que coadyuvan al desarrollo humano; y el segundo, la comprensión del concepto en los docentes de educación básica dentro de un escenario de profesionalización o actualización. La percepción de estas capacidades humanas en los procesos de aprendizaje de los docentes de educación básica en Tuxtla Gutiérrez, no había sido indagada como un fenómeno microregional que puede tener una influencia o impacto macroregional, por lo que se pretendió investigarla.

Para ello se considera en un polo, a nivel macroestructural, el perfil de desempeño del docente estipulado en el Marco para el Diseño y Desarrollo de Programas de Formación Continua y Superación Profesional para Maestros de Educación Básica en Servicio 2011-2012, donde se alude al desarrollo de capacidades humanas al considerar competente a un maestro frente a grupo si "Contribuye al desarrollo físico, social y emocional de los estudiantes, reconociendo la importancia de las distintas dimensiones del desarrollo humano, aplicando el mismo principio en su desarrollo personal integral" (Secretaría de Educación Pública, 2010, p. 14).

En otro polo, está el nivel microaccionalista, del cual se esperarían datos de la acción directa de los docentes. Sin embargo, sólo se conocen resultados calculados a partir de instrumentos como el índice de desarrollo humano (IDH) publicados en un boletín por el Instituto de Comunicación Social del Estado de Chiapas, en el que se menciona la "...tendencia positiva en los tres componentes del IDH: educación, salud e ingreso... el índice de educación ahora se obtiene mediante la escolaridad esperada y los años de escolaridad promedio para personas menores y mayores de 25 años, respectivamente." (Gobierno del Estado de Chiapas, 2012, marzo14).

Al conocer ambos polos, se hace pertinente el resolver junto con los maestros de educación básica, el problema de cómo se percibe el desarrollo de las capacidades humanas de nosotros mismos, para luego desarrollar y lograr las de nuestros alumnos, traduciéndose en desarrollo humano; con la consideración de las políticas de la SEP y los IDH publicados, respecto a la realidad de los alumnos y su tejido social en la microregión. También, cuáles son los intereses y factores condicionantes que afectan o intervienen en nuestro proceso de reflexión para el desarrollo de nuestras capacidades humanas. Del mismo modo, determinar cuáles son las capacidades humanas regionales, de nosotros los docentes en nuestro contexto local.

Dichos cuestionamientos en esta investigación, pueden acercarnos a la realidad socioeducativa regional en un contexto determinado, porque al saber -cómo deconstruir las capacidades humanas regionales de nosotros los docentes de educación básica en nuestros procesos de reflexión-, se puede comprender el potencial de cambio en nuestra acción docente ante la sociedad inmersa en una región. 


\section{Objetivos de estudio}

\section{Objetivo General}

Deconstruir conjuntamente con los participantes, las capacidades humanas regionales de nosotros los docentes de educación básica, en nuestros procesos de reflexión entre teoría y práctica, mediante la consideración de las condicionantes estructurales, en niveles macro y micro regionales, para identificar el potencial de cambio en nuestra praxis de docente como acción ante nuestros alumnos, quienes configuran el tejido social de Tuxtla Gutiérrez.

\section{Objetivos especificos:}

- Identificar la percepción de Capacidades Humanas en la intersubjetividad de nosotros los docentes en nuestro proceso de reflexión; respecto a: el perfil de desempeño del docente estipulado por la Secretaría de Educación Pública, los Índices de Desarrollo Humano en Chiapas y la realidad de los alumnos que integran el tejido social en la región socioeducativa en Tuxtla.

- Distinguir los intereses y factores condicionantes que afectan o intervienen en nuestros procesos de reflexión docente, para el desarrollo de nuestras capacidades humanas.

- Establecer las capacidades humanas regionales de nosotros los docentes, en diferentes situaciones dentro de nuestro contexto local.

\section{Metodología de investigación}

La perspectiva de investigación, se refiere a la posición del sujeto investigador en un programa, tradición o paradigma, que de acuerdo a los textos de Sandín Esteban (2004) y de Álvarez- Gayou Jurgenson (2005), se pueden identificar tres posturas emergentes en las últimas décadas para las Ciencias Sociales y Humanas: Positivista, con fundamentos en el positivismo lógico, considerado empírico-analítico, su finalidad en la investigación es explicar o verificar teorías; Sociocrítica, con raíz neomarxista y fundamentos en la teoría crítica, su finalidad en la investigación es identificar el potencial de cambio por medio de la reflexión o autocrítica, para emancipar sujetos; Interpretativa, con fundamentos en la fenomenología y hermenéutica, su finalidad en la investigación es comprender los significados de las acciones de las personas, para interpretar la realidad.

Al relacionar dichas posturas con el problema de investigación y los objetivos, se deduce que la postura teórica que se tuvo como sujeto investigador para el desarrollo de la investigación educativa realizada, junto con los maestros de educación básica en la región socioeducativa de Tuxtla, partió de la Teoría Crítica, considerada como núcleo o punto de fuga de la Perspectiva Sociocrítica, con raíz neomarxista; para identificar el potencial de cambio educativo por medio de la reflexión o autocrítica de nuestra praxis, en la región. 
Mediante esta perspectiva (ver Gráfico 1), relacionada al problema de investigación, se adoptaron complementariamente otras teorías que coadyuvaron a los objetivos del estudio regional, además de visualizar otros planos, como el filosófico, epistemológico y metodológico, del cual derivaron la aplicación de las técnicas y el diseño de los instrumentos.

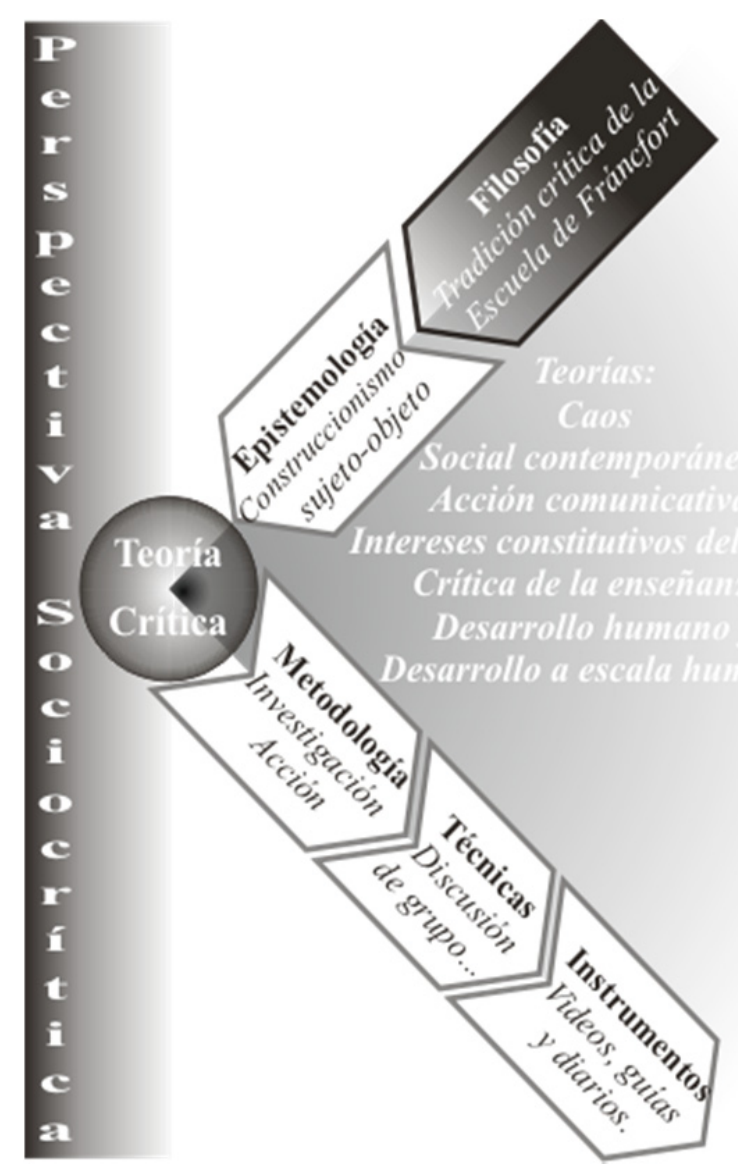

Gráfico 1. Perspectiva de la investigación educativa.

La metodología de la presente investigación educativa, estuvo orientada a la investigación-acción; porque que de acuerdo a los aportes que han realizado Carr y Kemmis (1988) a esta forma de investigar, se vislumbraba un camino para lograr el objetivo de esta investigación dentro del campo de los estudios regionales.

Estos autores, contribuyen junto con otros, en la identificación de tres modalidades de la investigación-acción, las cuales se relacionan directamente con los paradigmas actuales de la investigación educativa: técnica, enfocada al autocontrol de los docentes 
para el análisis de sus acciones en el aula; práctica, orientada a la reflexión de los maestros para ensayar las ideas de su praxis; y por último la modalidad que nos interesó, crítica, porque se centra en el aspecto social de la práctica educativa al integrar la acción y la estructura, para transformar las condiciones micro y macro establecidas.

La modalidad crítica de la investigación-acción realizada, condujo a que en el rol de investigadora se tuviera una actuación dentro de un grupo o aula como capacitador, en los cursos, para poder tener la oportunidad de proporcionar a los docentes de educación básica los instrumentos que develaron las distorsiones subyacentes en las interpretaciones generadas en torno al desarrollo de las capacidades humanas en la región Tuxtla Gutiérrez. En la intersubjetividad del espacio y tiempo de la investigación, se identificaron las contradicciones entre teoría y práctica las cuales se distinguieron como limitantes o condicionantes del cambio educativo (Sandín, 2004, p.163).

Ante esta metodología, la emancipación se podría dar en consecuencia, porque al definir conjuntamente las capacidades humanas necesarias de los docentes de educación básica, en sus procesos de reflexión, se pudo identificar el potencial de cambio en la praxis del docente ante los alumnos, quienes finalmente configuran el tejido social de Tuxtla Gutiérrez.

El resultado de la intersubjetividad emergente por la discusión en grupo; primeramente representó el potencial endógeno de cambio, en los procesos de reflexión del maestro de educación básica, refiriéndonos a un nivel micro; posteriormente mediante la responsabilidad que se adquiere, respecto a la práctica educativa en la región, se puede lograr transformar las estructuras socialmente establecidas en un nivel macro.

El posicionamiento en la teoría crítica con una perspectiva sociocrítica, marca la trayectoria a unas determinadas metodologías acordes, y que en este caso fue la de investigación-acción con modalidad crítica; ambas acotaron también, en una primer instancia, la selección de técnicas e instrumentos utilizados para la obtención de datos útiles al trabajo académico realizado.

\section{Técnicas e instrumentos de la investigación}

De acuerdo a las posturas planteadas con relación al objetivo de la investigación, se propuso para el desarrollo del estudio regional las siguientes técnicas e instrumentos, con la flexibilidad de ser cambiados o mejorados en la acción:

- Técnica de observación participante. Se realizó esta observación como investigador al interactuar implícitamente en el grupo objeto de estudio de la presente investigación.

- Los instrumentos utilizados fueron -videos y guías de observaciónporque facilitaron la obtención de datos de manera sistemática. 
- Técnica de entrevistas en profundidad. Considerada por el grupo Esbrina de la Universidad de Barcelona (Hernández, Sancho y Rivas, 2011); dicha técnica propició en esta investigación una cercanía a la realidad socioeducativa en el contexto de la región.

- Los instrumentos utilizados fueron-guías de entrevistas- las cuales se diseñaron con un nivel semiestructurado, para poder tener la flexibilidad que se requirió en este tipo de investigación.

- Técnica de discusión de grupo. Coadyuvó a comprender la realidad social, al producir una intersubjetividad en los espacios y tiempos de la interacción con los docentes participantes.

- Los instrumentos utilizados fueron -diarios de campo- que fueron elaborados conjuntamente entre con los docentes participantes; fueron semiestructurados para poder identificar situaciones condicionantes de la problemática en cuestión.

\section{Participantes}

En esta investigación educativa, los "otros" fueron los maestros de educación básica en servicio, correspondientes a los niveles de educación especial, preescolar, primaria y secundaria; pertenecientes al Centro de Maestros I-Tuxtla, que atiende o da cobertura a los dos subsistemas educativos en Chiapas: el Federal y el Estatal. Estos docentes fueron provenientes de centros escolares, dependientes de Supervisiones de Zona y Jefaturas de Sector ubicadas en la región Tuxtla. Es por ello que los procesos de actualización de dichos maestros se desarrollan en la ciudad capital de Chiapas. Siendo esta acción, favorable al proceso estratégico de la investigación (Ver Gráfico 2).

De acuerdo al proceso estratégico de la investigación, se obtuvieron participaciones de diversos docentes de educación básica de la región de estudio. En el Verano 2012, participamos 125 maestros del sistema estatal, del nivel secundaria con modalidad Telesecundaria, provenientes de la Zona Escolar 01 inmersa en el Sector Escolar 001. En el Verano 2013, participamos 12 maestros, pertenecientes a los sistemas federal y estatal, de niveles y modalidades distintas como Educación Especial, Preescolar, Primaria, Secundaria General y Telesecundaria. Finalmente, desde Septiembre 2014 hasta culminar con el proceso de estudio, participamos 7 docentes de ambos subsistemas, provenientes de los siguientes niveles y modalidades: Una participante de Educación Especial, dos de Primaria, dos de Secundaria General y dos de Telesecundaria. 


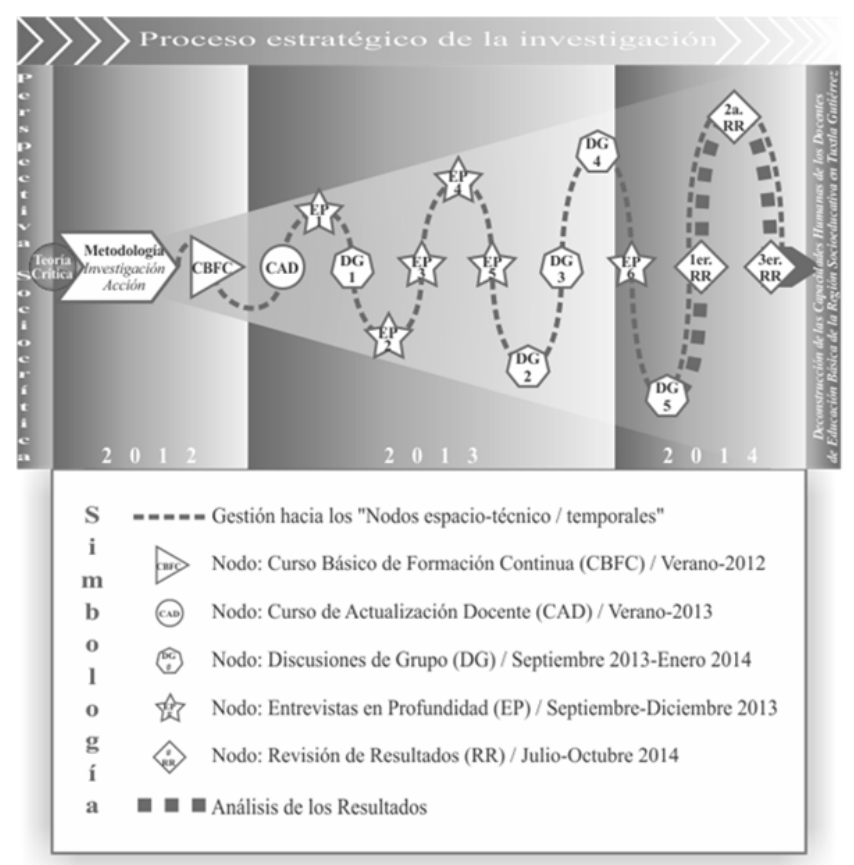

Gráfico 2. Proceso estratégico de la investigación.

\section{Resultados}

La investigación educativa, tuvo como resultado la deconstrucción de las capacidades humanas regionales de nosotros los docentes de educación básica, en nuestros procesos de reflexión entre teoría y práctica, en el que se consideraron las condicionantes estructurales, en niveles macro y micro regionales, con el alcance de haber identificado el potencial de cambio en nuestra praxis de docente como acción ante nuestros alumnos, quienes configuran el tejido social de Tuxtla Gutiérrez.

Los resultados de la investigación, objetivos y alcances, se concentran en el esquema (ver Gráfico 3); en el que se relacionan los significados que emergieron del discurso de los maestros participantes. De la parte inferior izquierda se inicia, al destacar como potencial de cambio a dos capacidades: reflexión y conciencia. La capacidad de reflexión, se relaciona directamente con la segunda columna que muestra la relación entre las capacidades humanas deconstruidas; ambas columnas se dirigen ascendentemente hacia la felicidad, por ser este el sentido de dichas capacidades, otorgado por los participantes. En la tercer columna, se mencionan más capacidades que directamente relacionadas con las anteriores, llevan a las consecuencias mencionadas en la cuarta columna; que al conjuntarlas culminan en la felicidad. Estas consecuencias, adquirieron significado hacia un beneficio individual y colectivo, representados ascendentemente sobre el extremo derecho. Y finalmente, mediante la 
dirección hacia la parte superior derecha, se resalta la relación y proyección de significados con el alumno inmerso en una región.

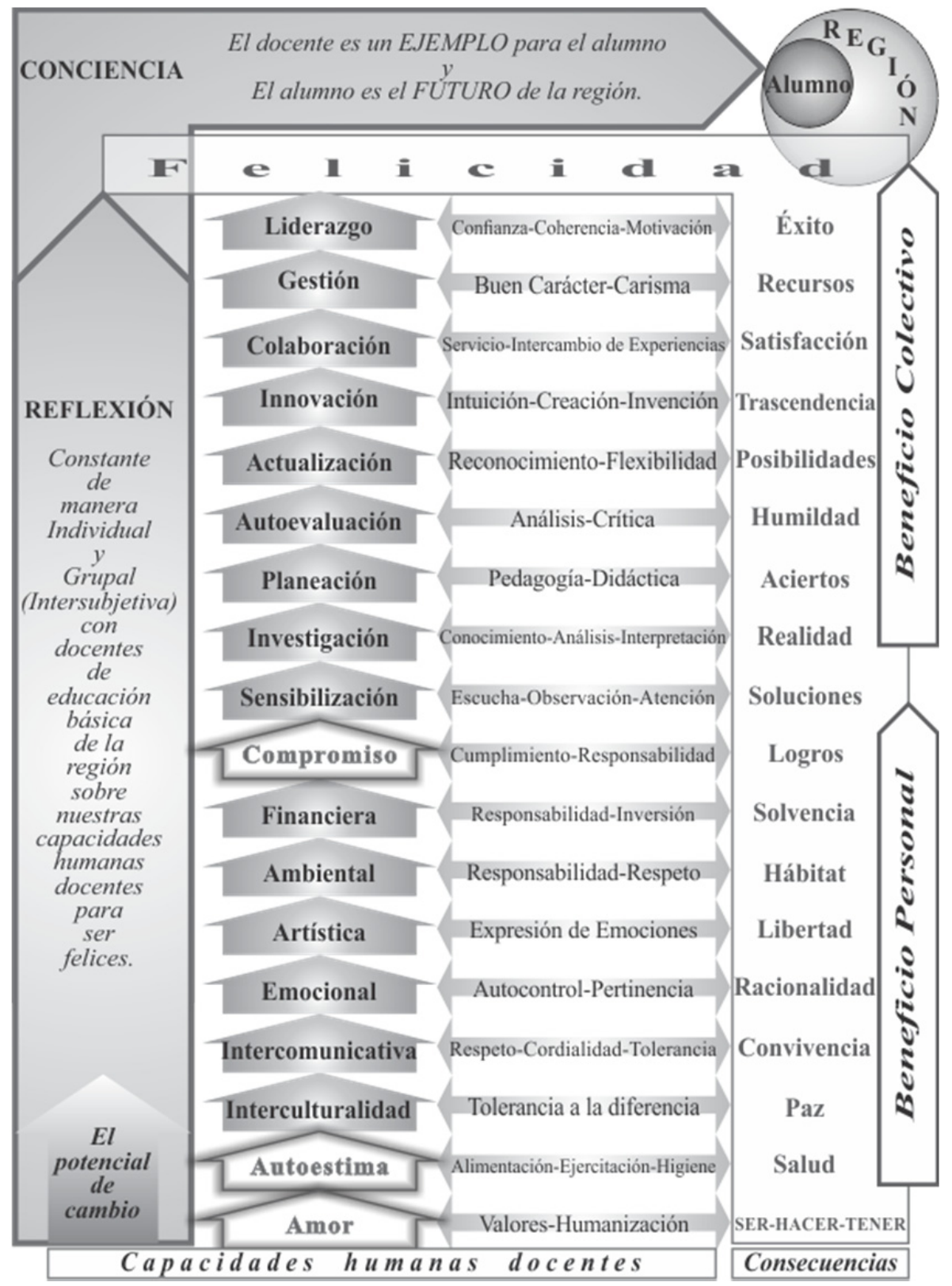

Grafico 3. Potencial de cambio y capacidades humanas de los docentes de educación básica en la región Tuxtla. 


\section{Discusión}

Al perseguir conjuntamente la deconstrucción de las capacidades humanas de nosotros los docentes de educación básica en una región socioeducativa como la de Tuxtla Gutiérrez, paralelamente se tuvo el alcance de identificar nuestro potencial de cambio en nuestra praxis de docente frente a nuestros alumnos, quienes pertenecen a familias y éstas a la misma región en la que estamos todos inmersos, tanto de manera individual como colectiva a la vez.

$\mathrm{Al}$ referirnos a nosotros los docentes, de manera individual, se responde al objetivo y alcance de la investigación, siendo las capacidades humanas de los docentes el foco de discusión; esto no quiere decir que se le esté otorgando una total responsabilidad, a únicamente los docentes, en la educación que se espera promueva una transformación regional. Lo que resulta de la investigación es una responsabilidad compartida con diversos actores de la educación, verbalizados por los docentes participantes, como lo son: alumnos, padres de familia, autoridades educativas y por supuesto los mismos docentes, que colectivamente pueden desarrollar nuevas formas de participación para la mejora de la propia región (Rivas, 2005).

En la intersubjetividad generada entre los docentes participantes, durante los tiempos y en los espacios diversos dentro del proceso de investigación-acción desarrollado, resultaron oportunidades para escuchar nuestras voces cargadas de experiencia, datos, ideas, cultura, en fin nociones intangibles pero con sentido regional, que nos acercaron a la comprensión de las condicionantes estructurales de nuestra práctica pero al mismo tiempo revalorarnos como humanos para rescatar y potenciar nuestra acción docente en nuestro contexto, con la posibilidad de cambiar dichas estructuras (Leite, 2005). Es por ello que a continuación se discuten cada uno de los datos deconstruidos conjuntamente.

\section{Potencial de cambio}

Al deconstruir la capacidades humanas, de los docentes, se tuvo el alcance de detectar, en el espacio y tiempo de la investigación-acción, el potencial de cambio de nosotros los docentes de educación básica en la región Tuxtla. Y dicho potencial se detona hacia el cambio regional por medio de dos capacidades que adquirieron relevancia en el proceso investigativo: la capacidad de reflexión y la capacidad de conciencia.

La capacidad de reflexión, como potencial de cambio para nosotros los docentes, se concibe como una acción primordial, constante o frecuente, acorde a las contribuciones de Dewey (2007); en esos tiempos, sobre y en la acción como lo aclara Schön (2011), respecto al desarrollo de nuestras capacidades humanas, al perseguir nuestra felicidad; también de dos maneras: una individual o subjetiva y otra grupal o intersubjetiva en tiempos y espacios compartidos con docentes de educación básica de la región.

Esto quiere decir, que el potencial de cambio docente, requiere en nosotros una reflexión continua con un posicionamiento rigurosamente crítico, tanto en lo interno que son nuestros pensamientos y lo externo que es la acción de verbalizar dichos 
pensamientos, sobre la razón y consecuencias del desarrollo o no de nuestras capacidades humanas, claramente expuestos en el discurso de los docentes participantes en la investigación-acción y en concordancia con las aclaraciones de Zeichner y Liston (1996).

En el proceso de investigación desarrollado mediante la observación paricipante, también salieron a la luz algunos datos que refuerzan a la reflexión como potencial de cambio: los pensamientos individuales al ser verbalizados adquieren un valor de representatividad o proyección de quien lo dice. Al escuchar los pensamientos de los demás inminentemente se genera una comparación crítica e individual sobre lo dicho por todos respecto a los propios. En todas las sesiones del proceso se observó la capacidad reflexiva que poseen, tanto de manera inmediata sobre lo verbalizado en una misma sesión, como de manera tardía pero con más reflejo de convicción sobre lo verbalizado en sesiones anteriores. Estos datos observados, sobre la reflexión de los docentes participantes, potencian un posible cambio en ellos mismos, sobre la discusión generada por los participantes.

Y a esto se le aunaría un indicio de cambio docente de mucha relevancia en lo observado: al inicio del proceso de investigación-acción, en la mayoría de los docentes se observó una actitud de reflexión sobre lo externo al individuo mediante construcciones discursivas dominantes y estructurales, las cuales nos dieron la mirada hacia las fuerzas macro y micro estructurales; después, en la medida en que avanzaba el proceso, las reflexiones que se desarrollaban de manera conjunta y generadas por la intersubjetividad, fueron llevando a los docentes a detectar la fuerza de su propia acción, en decir, que se trasladaban a una reflexión sobre lo interno, sobre ellos mismos, otorgándole sentido a su existencia y al ser feliz, indispensables para el siguiente potencial de cambio.

La capacidad de conciencia, se refiere específicamente en esta investigación a la conciencia sobre dos aspectos: una es que el docente es un ejemplo para el alumno y el alumno es el futuro de la región. Para llegar a ambos sentidos, la conciencia fue producto de una reflexión constante durante todo el proceso investigativo, tanto de manera interna o individual de cada maestro como de manera externa o grupal conjuntamente con los demás maestros.

Al inicio de la investigación todos tienen nociones de estos dos aspectos, los cuales algunas veces se verbalizan como reproducción de algo construido y aceptado en nuestros contextos educativos; pero al avanzar en el proceso de investigación-acción, estas nociones van adquiriendo otros matices que evidentemente son el resultado de una reflexión profunda de cada maestro.

Para llegar a una reflexión profunda, cada uno de los participantes ha trasitado muchas veces de lo exterior a su interior y viceversa, siendo la forma de llegar a determinar que nosotros los docentes necesitamos desarrollar una capacidad de conciencia sobre el impacto de nuestra acción ante nuestros alumnos; porque muchas veces reflejamos el no tener conciencia de ello. Este resultado de reflexión profunda, requiere en el docente como individuo, una introspección o conocimiento de sí, una 
reflexión sobre sí mismo en relación con su desempeño docente, trabajo o acción que finalmente lo constituyen en un ser humano docente.

El docente, al lograr desarrollar la capacidad de conciencia, sobre sí mismo, como un ser humano docente, descubre y encarna la idea de proyección de él mismo, viéndose como uno de los ejemplos próximos que tienen los niños que él atiende en su praxis docente. Esta concepción, ha sido claramente sustentada por Foucault (2005), en su libro Hermenéutica del sujeto, porque en sus resultados de investigación como en esta, las reflexiones nos llevan a comprender que existe entre las relaciones de maestro y alumnos, una proyección de cada sujeto inmerso y en especial la del maestro, como la ejemplar (Runge, 2003).

En este tenor, la conciencia de que los docentes representamos el ejemplo a seguir para el alumno, siendo este consciente de ello o no, finalmente nos lleva a un sentido de responsabilidad sobre lo que somos y hacemos, es decir al cuidado de sí. Es por esto, que en el proceso de la investigación, el discurso fue otorgándole sentido a la reflexión del docente sobre sus capacidades para ser feliz.

La felicidad del docente, adquirió significado en los mismos docentes cuando lo mencionaban en dos niveles: en un nivel propio, al mencionarlo como el objetivo o la razón de cualquier capacidad a desarrollar como individuos, con una reflexión que relaciona directamente la felicidad con el sentido de la existencia; en un nivel compartido, al mencionar la felicidad del docente proyectada hacia los que le rodean, siendo este el mejor ejemplo del ser docente, que traspasa una concepción de erudición o conocimiento de contenidos temáticos, sino algo más allá que configura al ser humano y docente.

Y finalmente en la conciencia del docente como ejemplo para el alumno, con tan solo la intención de ampliar la mirada, se deriva la conciencia de que este alumno, está en el presente y estará probablemente en el futuro en esta región. Esta conciencia, sobre el impacto o repercusión de nuestro ser envuelto en una sociedad, lleva la mirada a otros tiempos y a otros espacios, refiriéndonos no sólo al presente y al aula, sino más allá, al futuro y a la región. En conclusión se deriva una conciencia, sobre la proyección de uno mismo, primeramente en el alumno, quien a la vez se proyecta a su contexto familiar, luego este a un contexto vecinal, al grado de impactar a un contexto local o regional.

\section{Capacidades humanas}

Existe una línea que se dibuja y se desdibuja en las capacidades, entre humanas y docentes, porque se entiende que en el proceso de reflexión constante sobre nosotros mismos transitamos del "ser humano" al "ser docente", por lo que en momentos se separa y en otras se une en los discursos. Esto se ve reflejado en tres capacidades humanas de los docentes, que frecuentemente destacaron en la investigación de la siguiente manera: el "amor" es la base de todo, lo principal es la "autoestima" y sin el "compromiso" no se logra nada. Estas alusiones a lo primordial como capacidad 
humana docente antes de todas las demás, nos lleva a hacer un hincapié en ellas, sin soslayar la importancia que tienen las otras que conforman un todo.

En primer lugar, la capacidad de amor, adquiere sentido en diversos momentos en que los maestros le otorgan un significado; al referirse al amor como un valor o emoción interna e individual que se emana hacia el exterior por algo o alguien más y que de ese amor podrían derivarse otros valores o emociones que nos llevan al desarrollo de otras capacidades más. Parte de esta aportación es acorde a la de Maturana (2002) cuando menciona primordialmente a la emoción del amor para la constitución del dominio de actitudes y/o conductas que favorecen el escenario para la aceptación del otro para la convivencia y por ende para el fenómeno social.

Existe otra parte del discurso de los maestros referente a la capacidad de amar, en la que no solo se conciben en sociedad sino también en un fenómeno ambiental, al mencionar el amor y aceptación al otro pero también a algo, que en momentos alude a lo tangible y en otras a lo intangible, en algunos casos circunstanciales y otros conscientemente intencionales; como el amor a su trabajo y con ello el amor y la aceptación de las condiciones en que se desarrolle dicho trabajo, ya sean físicas o naturales. En estos sentidos, la capacidad de amar del docente es la base de su praxis, ineludible a fenómenos sociales y ambientales.

En segundo lugar, la capacidad de autoestima, nos transporta a diversas reflexiones sobre lo individual o interno del ser humano docente, antes de lo exterior ya sea social o ambiental. También, concatena con la capacidad de amar, pero esta vez a uno mismo; como muchas veces se mencionó en los discursos: primero debo amarme a mí antes de los demás, o, no puedo amar a los demás si no me amo a mí primero. Aunque en algunos momentos de las reflexiones sobre la autoestima se trasladaba a la del alumno y a la del docente, siempre salió a la luz la importancia de desarrollar dicha capacidad en el ámbito escolar.

La autoestima en el ámbito escolar, ha destacado entre las reflexiones finales de la investigación educativa de Blanco y Rodríguez (2014), con una relación estrecha con el conocimiento de sí, imprescindibles para el éxito escolar. Esta aportación junto con la de los docentes participantes en la investigación, otorga relevancia al proceso mismo de reflexión docente para el conocimiento y aceptación de sí, en un nivel interno sobre el desarrollo de la capacidad de autoestima, antes de un nivel externo.

$\mathrm{Y}$ en al referirnos a la capacidad de autoestima en un nivel interno, engloba varios aspectos del ser humano docente, derivados de los discursos como: la mente, el alma y el cuerpo de uno mismo. Es por ello que esta capacidad alude a la consciencia de sí, para la aceptación y el cuidado de sí; refiriéndose a lo que se le provee a nuestro propio ser por medio de todos nuestros sentidos, manifiestos en algunos casos con la alimentación, el ejercicio y el aseo, para ser saludable o tener salud y que evidentemente se proyecta hacia un nivel exterior.

Lo anterior, da coherencia a la reiterada aseveración de preponderancia a la capacidad de autoestima docente. Y que en el discurso docente dentro de un conocimiento de sí, se detonan otras capacidades a desarrollar como: la capacidad intercultural, que da cabida a la aceptación de la diferencia y en consecuencia la paz; la 
capacidad intercomunicativa, que mediante el respeto, cordialidad y tolerancia, se puede tener una convivencia; la capacidad artística, que manifiesta las más íntimas emociones de nuestro ser, otorgándonos libertad en la expresión; capacidad ambiental, que conlleva responsabilidad y respeto, en la conservación y continuidad de nuestro hábitat; y la capacidad fianciera, que también lleva consigo una responsabiliad para su inversión, para tener solvencia ante la cotidianidad del mundo en que vivimos.

En tercer lugar, la capacidad de compromiso, en las reflexiones docentes transitó de lo exterior a lo interior, esto se podría enteder como el tránsito entre el compromiso con lo exterior, nuestro trabajo docente, que requiere antes un compromiso con lo interior, nuestro sentido de existencia en el conocimiento de sí. Esto entrelaza las tres capacidades, la de amar, la de autoestima y la del compromiso, pero esta última se carga de sentido colectivo hacia la proyección regional o hacia lo exterior.

Este último sentido del compromiso docente, se comprende dentro de una construcción compartida, de acuerdo a las aportaciones de Rivas (2014) y en concordancia con las de los docentes participantes; porque el compromiso social que se tiene en la acción docente dentro de lo constituido como lo deseable del desempeño de un trabajo en la educación, inexorablemente alude al sentido de responsabilidad traducido como compromiso individual, dentro de un esquema moral interno de cada uno como ser humano y ser docente.

Por lo tanto, la capacidad de compromiso docente, lleva al cumplimiento del trabajo con sentido de responsabilidad, para finalmente lograr un verdadero cambio en nuestro contexto. Esto quiere decir que en la unión del compromiso social e individual, por convicción o moral, se permite el traspasar a otras capacidades que otorgan la posibilidad de la tan deseada transformación socioeducativa regional, como:

La capacidad de sensibilizarse, para saber escuchar, observar y atender a todo(s) lo(s) que nos rodea(n) y así detectar problemas y acercarnos más a sus posibles soluciones; la capacidad de investigar, para conocer, analizar e interpretar, la realidad; la capacidad de planear, con pedagogía y didáctica, para acercarse más a los aciertos; capacidad de evaluarse, de manera constante, analítica y crítica, haciéndonos más humildes; la capacidad de actualización, mediante el reconocimiento de las debilidades o carencias, con actitud flexible, dando cabida a innumerables posibilidades en la acción docente; la capacidad de innovar, al intuir, crear e inventar, para trascender; la capacidad colaborativa, que al compartir experiencias y servir al otro, se obtiene una satisfacción; la capacidad de gestión, mediante un buen carácter y carisma, facilite la obtención de diversos recursos; y por último la capacidad de liderazgo, que requiere confianza en los demás, coherente con lo que se piensa, se dice y se hace, y así motivar al cambio, traducido como éxito docente.

$\mathrm{Y}$ en conjunto, el desarrollo de todas las capacidades mencionadas, de acuerdo a las aportaciones derivadas de las reflexiones de los docentes, no tendrían ningún sentido si no se persigue la propia felicidad. Esto hace de inmediato un hilo conductor con la teoría de Sen (2000) y Max-Neef (2010), porque en la práctica, estas dependen de la propia escala de valores que le otorga cada uno de los docentes al perseguir su felicidad. 


\section{Beneficio personal y colectivo}

Existen diversas construcciones sociales ya preestablecidas sobre los conceptos de beneficio personal y beneficio colectivo, las cuales se fueron transformando con las constantes reflexiones dentro del proceso investigativo. Al reflexionar sobre las capacidades humanas de los docentes y detectar el potencial de cambio, con una conciencia profunda del ser humano y docente a la vez, ineludiblemente se ha transitado entre las consecuencias de la acción docente sobre lo personal y sobre lo colectivo.

Reflexión escrita y anónima del Verano-2012:

La práctica docente no es un acto aislado o individual, debe transmitir contenidos, desarrollar pensamientos, procesos de contenido, además propone formar alumnos capaces de responder a nuevas exigencias y a enfrentar los constantes cambios sociales que ocurren dentro de su comunidad (2012).

En los discursos, se hacían alusiones de las consecuencias positivas, pero también negativas, de la acción docente; que en su sentido más estricto serían las consecuencias del desarrollar o no las capacidades humanas que emegieron en la intersubjetividad generada en el proceso. Es evidente que las consecuencias positivas se traducen en beneficios, pero lo que aquí se destaca como importante en la reflexión y conciencia de los docentes, es el tránsito de lo personal a lo colectivo.

Como parte de los resultados de la investigación, con la consideración previa de que cada participante tiene en sí su propia escala de valores ante los discursos emitidos, lo que se ha generado en la intersubjetiviad, es la concepción de nuestra acción docente con dos efectos que deben ser simultáneos e inseparables: el beneficio personal y el beneficio colectivo, que de acuerdo a la conciencia o escala de valores e intereses de cada docente, le provee felicidad; esto puede permearse sobre una nueva cultura de la organización escolar, al concebirse en colectivo (Rivas, 2003).

\section{Docente-alumno-región}

La deconstrucción de nuestras capacidades humanas, la identificación de nuestro potencial de cambio en la reflexión y conciencia, más el concebirnos en colectivo, abre la mirada al tejido social que forman nuestros alumnos en una región y lleva a atender la acción que tenemos ante ellos, si aspiramos a una tranformación socioeductiva regional.

Reflexión escrita y anónima del Verano-2012:

Tenemos la finalidad, de tener ante todo la docencia, para enfrentar las futuras generaciones que son las que sacarán adelante el pueblo, el país, etc., no es nada fácil el ser docente, porque enfrentamos una gran responsabilidad ante la población estudiantil (2012). 
En esta investigación, los docentes participantes con su corta o larga experiencia en la región, verbalizaron en diferentes momentos y escenarios el cómo se ha visto reflejada la acción docente en las distintas generaciones de la educación básica en Tuxtla, refiriéndose a las microregiones que la conforman. Esto ha dado cabida, al resultado de llevar la mirada más amplia sobre el impacto positivo o negativo de la acción docente dentro de la relación alumno y región, con la posibilidad de un futuro mejor.

\section{Conclusiones}

Con los resultados de la investigación educativa desarrollada, haciendo referencia a los objetivos y alcances que se dejan ver y plasmar entre letras, como los que no están escritos por no ser el foco investigativo y que pudieran ser semillas para otras investigaciones, por todos ellos, se concluye en varios aspectos, desde generales a particulares.

El más amplio o general, es la prospectiva de desarrollo de futuras investigaciones educativas, porque al posicionarnos dentro del campo de los estudios regionales y con visión transdisciplinar; nos acercamos al conocimiento y comprensión de nuestras realidades locales o microregionales, sin soslayar lo macroregional, al poner en tensión las fuerzas estructurales y las de la acción; porque tanto el problema, discusiones, reflexiones y soluciones, son propios de nuestro contexto. Por ende se adquiere un sentido mayor de apropiación y responsabilidad ante una posible transformación regional.

De esto se deriva otra conclusión, existe un potencial endógeno y exógeno en las regiones, pero al mismo tiempo con limitantes estructurales. Los participantes en la investigación se mostraron ávidos de nuevas formas de participación social y educativa porque el desarrollo de la investigación-acción facilitó el escenario y la apertura para escuchar nuestras voces, que pusieron a la luz la realidad local y en consecuencia el detectar nuestro potencial de cambio. Pero también quiere decir, que tenemos la necesidad de espacios y tiempos para la reflexión participativa hacia la mejora de situaciones regionales.

En las conclusiones más particulares, la reflexión de manera conjunta, amplía la mirada de los participantes, mediante la confrontación constante entre teoría y práctica, entre lo socialmente ya construido y lo nuevo por construir, entre lo exterior y lo interior al ser humano; esto incita a la reflexión individual, interna y profunda en el ser humano, ante sus propios intereses y escala de valores para darle sentido a su existencia, antes de poder concebirse en sociedad o en colectivo; para así sentirse partícipes en una región.

Y finalmente, se concluye en que las capacidades humanas y potencial docente, deconstruidos conjuntamente con los docentes participantes, son la respuesta en un determinado tiempo y espacio; estos resultados de ninguna manera se conciben como hegemónicos o la verdad última, más bien exponen ante nosotros los participantes en el 
proceso investigativo y en quien lea estas líneas, posibilidades de acción docente hacia la tan deseada transformación socioeducativa en las regiones.

\section{Referencias bibliográficas}

ÁlVAREZ-GAYOU JURGENSON, J. L. (2005). Cómo hacer investigación cualitativa, fundamentos y metodología. México: Paidós.

BECK, U. (2008). Lógicas, dimensiones y consecuencias de la globalización. ¿Qué es la globalización?, 73-132. Barcelona: Bolsillo Paidós.

BLANCO, N. Y RODRÍGUEZ, C. (2014). La actitud y el compromiso hacia la escuela en estudiantes de secundaria de éxito escolar. Apuntes del Seminario Política Curricular y Prácticas Educativas. Málaga, España: DEC-UMA.

BONAL, X. (2002). Globalización y política educativa: un análisis crítico de la agenda del Banco Mundial para América Latina. Revista mexicana de investigación educativa, 64 (3), 3-35.

CARR, W Y KEMMIS, S. (1988) Teoría crítica de la enseñanza. La investigaciónacción en la formación del profesorado. Barcelona: Martínez Roca.

DEWEY, J. (2007). Cómo pensamos. Barcelona: Paidós Ibérica.

FERRERO, M. (Mayo de 2006). La glocalización en acción: Regionalismo y paradiplomacia en Argentina y el Cono Sur Latinoamericano. Integración en Ideas. IDELA/UNT.

FOUCAULT, M. (2005). La hermenéutica del sujeto. México: Akal.

GOBIERNO DEL ESTADO DE CHIAPAS. (14 de Marzo de 2012). Desarrollo humano, pilar en el avance histórico de Chiapas en la lucha contra la pobreza: PNUD. Recuperado el 18 de Junio de 2012, de Sala de Prensa del Poder Ejecutivo: http: //www.chiapas.gob.mx/prensa/boletin/desarrollo-humano-pilar-en-el-avancehistorico-de-chiapas-en-la-lucha-contra-la-pobreza-pnud.

HERNÁNDEZ, F., SANCHO, A. M. y RIVAS, J. I. (2011). Historias de vida en educación. Biografías en contexto. Barcelona: ESBRINA-RECERCA.

IXTACUY LÓPEZ, O. (2012). Región, geografía y economía. Antología del Seminario General de Estudios Regionales. Tuxtla Gutiérrez, Chiapas, México: DER-UNACH.

LEITE, A. (2005). La educación en escenarios latinoamericanos posibles: el difícil equilibrio entre lo cotidiano y lo global. Revista Electrónica Save the Children.

MARTÍNEZ-PELLÉGRINI, S. (2003). Convergencia regional e integración: los casos de México y España. En N. A. Fuentes Flores, A. Díaz-Bautista, S. E. MartínezPellégrini, \& E. C. Norte (Ed.), Crecimiento con convergencia o divergencia en las regiones de México (págs. 133-149). México, D. F.: Plaza y Valdés. 
MATURANA, H. (2002). Emociones y lenguaje en educación y politica. Chile: Dolmen.

MAX-NEEF, M. A. (2004). Fundamentos de la transdisciplinariedad. Valdivia, Chile: Universidad Austral de Chile.

MAX-NEEF, A. M., ELIZALDE, A. Y HOPENHAYN, M. (2010). Desarrollo a escala humana: Opciones para el futuro. Madrid: Biblioteca $\mathrm{CF}+\mathrm{S}$.

MC CAULEY, B. (Marzo de 2006). Los Estudios Territoriales de la OCDE: La Región Mesoamericana - El Sureste de México y América Central. Obtenido de Estudios Territoriales de la OCDE: la Región Mesoamericana: http://www.oecd.org/documentprint/0,3455,es_36288966_36288607_36612171_1_ 1_1_1,00.html (PDF).

RIVAS, J. I. (2003). La perspectiva cultural de la organización escolar: marco institucional y comportamiento individual. Revista Educar, Barcelona.

RIVAS, J. I. (2005). Nuevas formas de participación. VIII Congreso Estatal de voluntariado: Compartiendo solidaridad. Granada, España: Consejería Bienestar Social de Andalucía.

RIVAS, J. I. (2014). Cultura institucional y compromiso moral en el trabajo del profesor. Apuntes del Seminario Políticas de Innovación en Organización y Gestión de Centros Educativos. Málaga, España: DEC-UMA.

RUNGE PEÑA, A. C. (2003). Foucault o de la revaloración del maestro como condición de la relación pedagógica y como modelo de formación. Una mirada pedagógica a la hermenéutica del sujeto. Revista Educación y Pedagogía.

SÁNCHEZ JUÁREZ, I. L. (2011). Calidad institucional, desaceleración del crecimiento y subdesarrollo en México. En L. E. Gutiérrez Casas, \& M. Limas Hernández, Nuevos Enfoques del Desarrollo. Una Mirada desde las Regiones (págs. 48-101). Ciudad Juárez, Chihuahua, México: Universidad Autónoma de Ciudad Juárez Red Iberoamericana de Estudios del Desarrollo.

SANDÍN ESTEBAN, M. P. (2004). Investigación Cualitativa en Educación. Fundamentos y Tradiciones. Madrid: Mc Graw Hill.

SCHÖN, D. A. (2011). The reflective practitioner: how professionals think in action. Aldershot: Ashgate.

SECRETARÍA DE EDUCACIÓN PÚBLICA. (2010). Marco para el diseño y desarrollo de Programas de Formación Continua y Superación Profesional para Maestros de Educación Básica en Servicio 2011-2012 . México, D. F.: Dirección General de Formación Continua de Maestros en Servicio de la Subsecretaría de Educación Básica.

SEN, A. K. (2000). Desarrollo y libertad. Madrid: Planeta. 
TOURAINE, A. (2005). Los derechos culturales. En A. Touraine, Un nuevo paradigma para comprender el mundo de hoy (págs. 181-227). Barcelona: Paidós Estado y Sociedad 135.

ZEICHNER, M. y LISTO, P. (1996). La práctica docente reflexiva. Raices históricas de la enseñanza reflexiva. Obtenido de: http://upvv.clavijero.edu.mx/ cursos/observacion_practica_reflexivaII/programa/documentos/Zeichner.pdf.

\section{Correspondencia con la autora}

Irán Guadalupe RODRÍGUEZ RAMÍREZ

Privada Pirita Sur 144, Fraccionamiento San Fernando

Tuxtla Gutiérrez,

29010 Chiapas

México

e-mail: iran.rodriguez@hotmail.com 